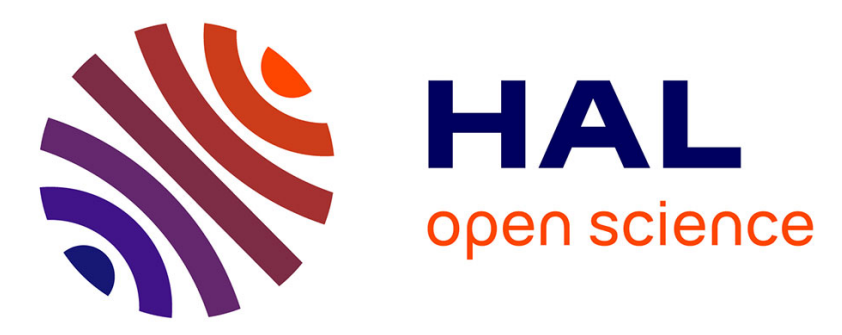

\title{
Fault Tolerant Control of a Teleoperated Piezoelectric Microgripper
}

\author{
Moussa Boukhnifer, Antoine Ferreira
}

\section{To cite this version:}

Moussa Boukhnifer, Antoine Ferreira. Fault Tolerant Control of a Teleoperated Piezoelectric Microgripper. Asian Journal of Control, 2013, 15 (4), pp.1-13. 10.1002/asjc.593 . hal-00821623

\section{HAL Id: hal-00821623 \\ https://hal.science/hal-00821623}

Submitted on 22 May 2013

HAL is a multi-disciplinary open access archive for the deposit and dissemination of scientific research documents, whether they are published or not. The documents may come from teaching and research institutions in France or abroad, or from public or private research centers.
L'archive ouverte pluridisciplinaire HAL, est destinée au dépôt et à la diffusion de documents scientifiques de niveau recherche, publiés ou non, émanant des établissements d'enseignement et de recherche français ou étrangers, des laboratoires publics ou privés. 


\title{
Fault Tolerant Control of a Teleoperated Piezoelectric Microgripper
}

\author{
Moussa Boukhnifer ${ }^{1}$ and Antoine Ferreira ${ }^{2}$ \\ ${ }^{1}$ Laboratoire Commande et Systèmes \\ ESTACA - École d'Ingénieurs \\ 34 rue Victor Hugo - 92300 Levallois-Perret, France \\ ${ }^{2}$ Laboratoire PRISME, ENSI de Bourges \\ 88 bd Lahitolle, 18020 Bourges Cedex, France
}

\begin{abstract}
This paper presents a new architecture of control for micro-teleoperation system using a fault tolerant control (FTC) strategy to compensate for the degradation effects of piezoelectric-based microgipping system. The proposed strategy uses passivity approach for the bilateral controller and robust fault tolerant control (FTC) for the two-fingered microgripper system. First, the bilateral controller architecture uses the passivity approach for the teleoperation system in the presence of time delay and scaling factor variations. Second, the FTC controller is designed in such a way that the performance and robustness may be done separately which has the potential to overcome the conflict between performance and robustness in the traditional feedback framework. The controller architecture works in such a way that the feedback control system will be solely controlled by the $P I$ performance controller for a nominal model and the robust $H_{\infty}$ controller will only be active in the presence of uncertainties or degradation disturbances. The experimental and simulation results show clearly the effectiveness of the proposed approach against time delays, scaling factors and fault disturbances.
\end{abstract}

\section{INTRODUCTION}

The two major conflicting issues in scaled teleoperation are stability and transparency performances. The majority of the research adopts passivity concepts to ensure stability in the presence of time-delay and scaling force factors. Anderson and Spong [1] derived a control law based on passivity and scattering theory to ensure teleoperative stability subject to any time delay, but performance was shown to degrade as the time-delay was increased. Niemeyer and Slotine [2] also proposed an approach based on passivity and scattering theory to address time-delay in teleoperation. The choice of the scaling gain to properly reshape the master-slaveenvironment is still a remaining problem. The transparency is altered since the reshaped microenvironment looses its dynamic character. To solve this problem, Park [3] has introduced a velocity-force scaling property which happens at micro-teleoperation and used modified 4-channel architecture (4C) control, originated by Hashtrudi-Zaad et al.[4]. Based on these haptic coupling schemes, passivity controllers have been adapted to microscale in order to preserve long-range attraction forces such as van der Waals interactions, or capillary effects [5],[6]. Furthermore, it can satisfy passivity conditions for optimized-transparency in micro-teleoperation systems handling small inertial microobjects with nonlinear stiffness [7].

Recently, reliable and safer microsystems are growing in micromanipulation (biological cells, MEMS microparts, optical microcomponents) due to complex and various unexpected components faults and degradations (actuators, sensors) [8],[9]. From the authors's knowledge, robust bilateral control of micromanipulation systems in the presence of the faulty sensors or actuators have not been addressed. In this paper, we extend our previous work on wavebased bilateral micro-teleoperation [10] to the case of faulttolerant control of a piezoelectric-based microgripping system. The problem of designing a robust and passive fault tolerant controller (FTC) is addressed using wave variablesbased theory. In order to satisfy passivity condition for optimized-stability, all parts of the bilateral teleoperation system have to be strictly passive: master (operator-haptic interface-controller), bilateral communication channel and slave (controller-microgripper). Thus, a successful fault tolerant strategy is to be stable and transparent in non-faulty operating conditions (optimized-stability and -transparency) and to be robust by handling the variation and/or degradations in the micromanipulation system (slave part). Such approach can be designed through controller adaptation [11], reconfiguration [12] or switching [13]. The proposed FTC approach falls in the second category where a standard Youla parameterization is used for the FTC controller architecture. Compared to existing robust FTC designs based on sliding mode control [14], optimal control [16], neuro control [17] [18], fuzzy robust control [19], or based on robust multicontroller structure [15], the Youla parameterization allows the design of a new FTC controller architecture with a clear separation between performance and robustness [20]. The slave controller architecture works in such a way that the feedback control system will be solely controlled by the $P I$ performance controller for a nominal model and the $H_{\infty}$ robustification controller will only be active in the 
presence of uncertainties or degradations. The simulation and experimental results demonstrate a clear separation between optimized-transparency in the former case and optimizedrobustness in the later case.

The paper is organized as follows. In Section 2, the wavebased controller architecture subjected to time-delays and scaling factors is presented. In section 3, we recall the standard Youla parameterization before to introduce the proposed FTC control architecture in section 4. Finally, simulation and experimental results are presented in order to prove the effectiveness of the proposed approach.

\section{BACKGROUND ON PASSive Bilateral CONTROL OF MiCRO-TELEOPERATION SYSTEM}

\section{A. Wave-Based Bilateral Control}

Detailed specifications of the wave-based microteleoperation system are discussed in [10]. A brief summary is given here. A bilateral telemicromanipulation system is modeled with linear approximation consisting of a series of functional blocks, as shown in Fig.1, composed mainly of the human operator (block1), the controlled scaled bilateral telemanipulator (block 2), and the working microenvironment (block 3). The block 1 is composed of a local hand controller $G_{m}$ represented by the haptic interface $P_{m}(s)$ (master) and its velocity controller $K_{m}$ and a human operator (modeled by an impedance $Z_{h}$ ) which applies a force $F_{h}$. The block 2 represents the wave-based communication channel $K$ where $e^{-s T_{1}}, e^{-s T_{2}}$ represent the time-delays and $k_{p}, k_{f}$ are the scaling factors for the position and the force, respectively. The block $3 G_{s}$ represents the micromanipulator $P_{s}(s)$ (slave) and its velocity controller $K_{s}$ interacting with the microenvironment $Z_{e}$ via a force $F_{e}$. The interaction force with the environment $F_{e}$ measured by the microgripper is sent back to the operator as a reference for the haptic interface such that $f_{h}=k_{f} \cdot f_{e}$.

The transfer functions of master $P_{m}(s)$ and slave $P_{s}(s)$ are given by the following equations:

$$
P_{m}(s)=\frac{1}{m_{m} s^{2}+k_{m} s+b_{m}} P_{s}(s)=\frac{1}{m_{s} s^{2}+k_{s} s+b_{s}}
$$

where $m_{m}=10, m_{s}=4.88$ are respectively the mass of the master and slave ; $k_{m}=0, k_{s}=0.515 .10^{5}$ are damping coefficients and $b_{m}=0, b_{s}=0.232 .10^{8}$ are spring coefficients.

The bilateral architecture of Fig.1 can be viewed as the connection of individual blocks $G_{m}, G_{s}$ and $K$. If individual blocks of the network model are passive, the overall system is passive, and it is sufficient to make the system stable [21]. In the case of master-slave systems, if we could assume that the operator and the environment are passive systems, then the sufficient condition of stability is that the teleoperator two-port channel itself must be passive [22],[23]. Strictly speaking, however, the operator is not passive he/she has

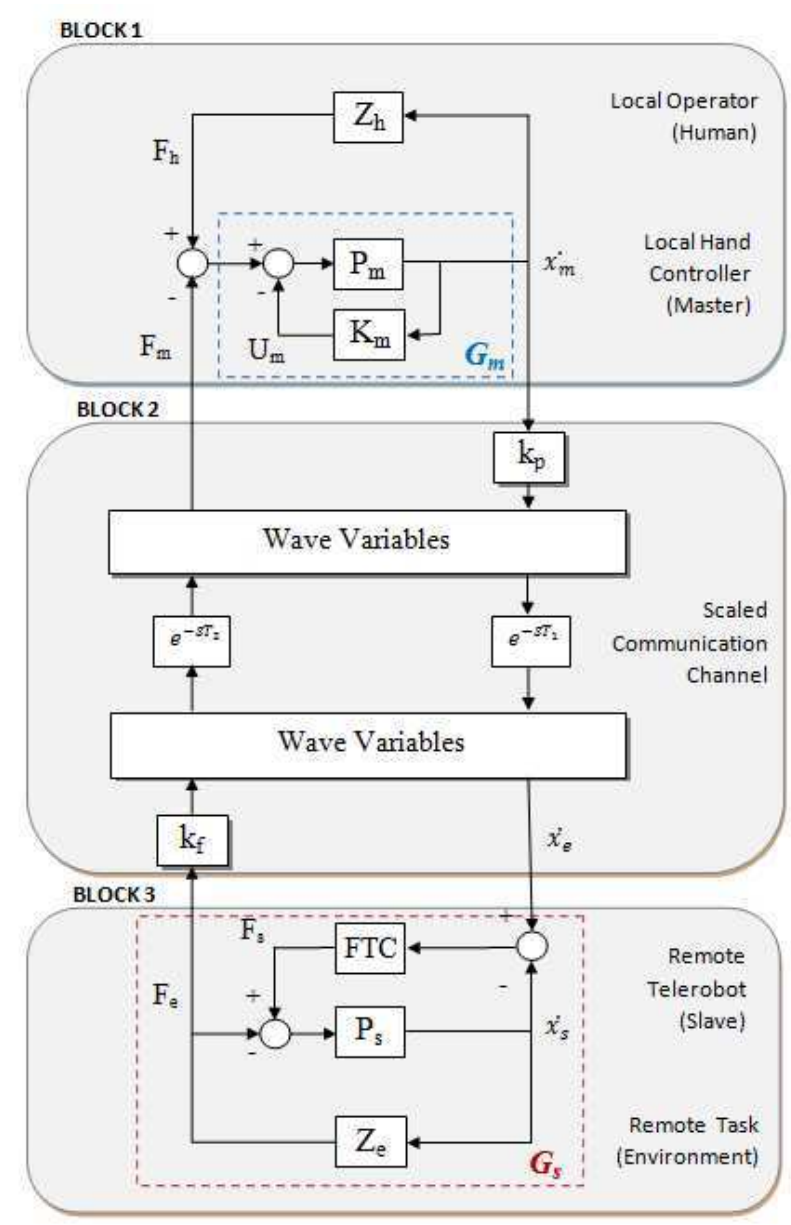

Fig. 1. Wave bilateral controller with FTC slave controller for a force reflecting micromanipulator.

muscles as the power source. Furthermore, assuming an exact and linear time invariant (LTI) model of an environment at the microscopic scale is generally a tenuous presumption due to nonlinear effects, most notably surface tension based capillary forces in humid environments and electrostatic forces in dry environment which acts as active sources. The passivity of traditional teleoperators is very sensitive to transmission delays which can destroy passivity of the entire system by altering the power flow in the system and generating excess energy. In addition, scaled teleoperators face to scaling problems of nonlinear positive gains matrices (scaling position and force factors) that preserves its passivity only if gains are equal, which is hard to find in real micromanipulation system. In order to prove the passivity of the overall system, it is required to prove the passivity of the three parts, that is, the human operator-local hand controller, the time delayed scaled communication channel and the environment-slave controller. To guarantee stability in presence of time delay and scaling factors, operator and 
environment uncertainties, wave variables transformations [24] and dissipativity concepts [25] are used in this study.

First, let us define the power entering in the system of communication as:

$$
P_{i n}=\dot{x}_{m}^{T} F_{m}-\dot{x}_{e}^{T} F_{e}
$$

where $\dot{x}_{m}, \dot{x}_{e}$ are respectively the velocities of the haptic interface and the microgripper. The waves variables $\left(u_{e, m}, v_{e, m}\right)$ are defined as follows:

$$
\begin{cases}u_{m}=\frac{b \dot{x}_{m}+F_{m}}{\sqrt{2 b}}, & u_{e}=\frac{b \dot{x}_{e}+F_{e}}{\sqrt{2 b}} \\ v_{m}=\frac{b \dot{x}_{m}-F_{m}}{\sqrt{2 b}}, & v_{e}=\frac{b \dot{x}_{e}-F_{e}}{\sqrt{2 b}}\end{cases}
$$

where $b$ is a characteristic wave impedance of the transmission line which effects the overall system behavior as described below. Its choice is crucial since it will be tuned to realize the matching impedance between the communication block and the haptic interface. The return of unexpected waves is then eliminated. The power transfer can also be rewritten as follows :

$P_{i n}=\dot{x}_{m}^{T} F_{m}-\dot{x}_{e}^{T} F_{e}=\frac{1}{2}\left(u_{m}^{T} u_{m}-v_{m}^{T} v_{m}+v_{e}^{T} v_{e}-u_{e}^{T} u_{e}\right)$

From (4), the controlled telemanipulator part that consists of the master manipulator and the slave manipulator is passive against the human operator and the environment, then the whole system is stable in view of passivity.

\section{B. Passivity of Human-Master Dynamics}

In general, when the operator carries out a task by using a telemanipulation system, we assume that the operator does not make the whole system unstable on purpose, and in addition, the operator is passive against an external input [26]. It follows that the master hand controller with its local force feedback controller ( $P I$ controller) is passive against the human operator when force feedback controller values are chosen adequately in order to passivate the human-master dynamics.

\section{Passivity Formalism with Scaling Factors}

In order to prove the passivity of the proposed scaled telemanipulation system with scaling factors $k_{p}$ and $k_{f}$, we will rewrite the constraints in the waves space and derive conditions between the parameters. The scaling factors represented by the gains $k_{p}$ and $k_{f}$ :

$$
\left\{\begin{array}{l}
\dot{x}_{e}=k_{p} \dot{x}_{m} \\
F_{m}=k_{f} F_{e}
\end{array}\right.
$$

As each system is expressed in the power variables $(\dot{x}, F)$, it can be written in terms of the wave variables $(u, v)$ which are useful to convert dimensional scaling factors from

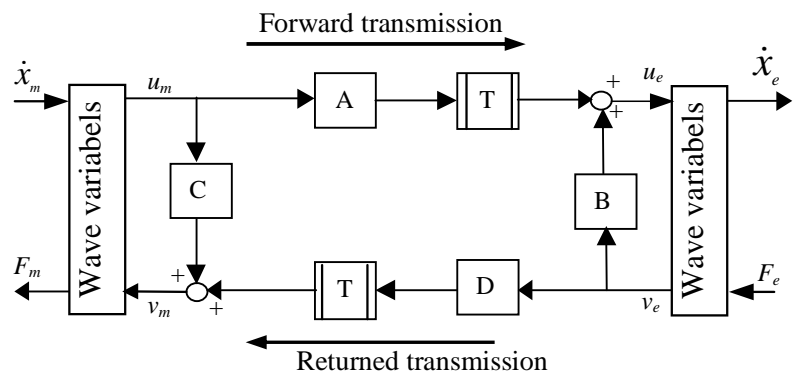

Fig. 2. Equivalent diagram of scaling factors in wave domain.

one domain to another. Let us consider the following bilateral controller expressed in the waves space (Fig.2) where $A, B, C, D$ are the transfer of the scaling factors $k_{p}$ and $k_{f}$ in the wave domain in presence of the delays $T$.

The scaling factors can be expressed as follows:

$$
\left(\begin{array}{c}
u_{e} \\
v_{m}
\end{array}\right)=\left(\begin{array}{cc}
A & B \\
C & D
\end{array}\right)\left(\begin{array}{c}
u_{m} \\
v_{e}
\end{array}\right)
$$

For the scaling factors $k_{p}$ and $k_{f}$, we obtain in the wave domain the following forms:

$$
\left\{\begin{array}{l}
A=\frac{2 k_{p}}{\left(1+k_{p} k_{f}\right)}, \quad B=\frac{k_{p} k_{f}-1}{1+k_{p} k_{f}} \\
C=\frac{1-k_{p} k_{f}}{1+k_{p} k_{f}} \quad D=\frac{2 k_{f}}{\left(1+k_{p} k_{f}\right)}
\end{array}\right.
$$

The telemicromanipulation system preserves its passivity, if and only if, the scaling factors are equal $\left(k_{p}=k_{f}\right)$. When considering different scaling factors $k_{p} \neq k_{f}$, the interconnections $A, B, C$ and $D$ should be redefined with respect to the manipulator-environment interaction at the micro-scale. In order to ensure the passivity of our system in presence of time-delays, we introduced the impedance filters $G(s)$ and $F(s)$ after each delay block. The system is passive, if and only if, the following three conditions are satisfied [10]:

$$
\left\{\begin{array}{l}
|F(s)|^{2} \leq \frac{k_{f}}{k_{p}} \\
|G(s)|^{2} \leq \frac{k_{p}}{k_{f}} \\
\left(k_{f} k_{p}+1\right)^{2}\left(k_{p}|F|-k_{f}|G|\right)^{2}-\left(2 k_{f} k_{p}\right)^{2}(\mid G \cdot F-1)^{2} \mid \leq 0
\end{array}\right.
$$

\section{Passivity of Slave-Environment Dynamics}

The environment part is represented in Fig.1 by a block $G_{s}$ composed of the environment with the negative feedback of slave and the local force feedback controller. These subsystems cannot be simply considered as two passive subsystems in parallel, so it is necessary to ensure the passivity of the all parts, i.e., the block $G_{s}$ in the presence of the faulty actuator and/or sensor. Various control strategies were tested by the authors. In [10], a high-performance PI controller ensured passivity for a nominal model with very little robustness against a class of possible faults in sensors and/or 
actuators (model uncertainties and external disturbances). For this reason, a worst-case $H_{\infty}$ controller [27] was tested in order to increase the robustness but at the expense of performances (i.e., lack of force transparency). This potential conflict between performance and robustness has been solved using the Youla controller parameterization proposed in [11]. We synthesized a passive controller for the slave/environment model $G_{s}$ in such a way that the feedback control system will be solely controlled by the $P I$ performance controller for a nominal model and the $H_{\infty}$ robustification controller will only be active by assuming the possible actuators and/or sensors failures as model uncertainties. In the both cases the passivity must be verified using the FTC controller architecture.

\section{Fault Tolerant Control Design of MICROGRIPPER}

\section{A. FTC Controller Architecture}

In this section, we use the Youla parameterization in a nontraditional way and we explain how to use this architecture in order to ensure both performance and robustness outcomes [11]. Firstly, we consider the feedback diagram presented in Fig.4. The reference signal $r$ enters into the system from a different location. Nevertheless, the internal stability of the system is not changed since the transfer function from $y$ to $u$ is not changed. Thus, this controller implementation also stabilizes internally the feedback system with plant $P_{0}$ for any $Q \in H_{\infty}$ such that $\operatorname{det}(\tilde{V}(\infty)-Q(\infty) \tilde{N}(\infty)) \neq 0$. Due to the similarity with the well-known internal model controller (IMC) given by the figure Fig.3, see [28] for details, the controller framework of the Fig.4 is referenced by [11] as a generalized internal model control (GIMC). We see the similarity on their connections and the possible advantages of new GIMC over the traditional IMC where the distinguished feature of this controller implementation is that the inner loop feedback signal $f$ is always zero, i.e., $f=0$, if the plant model is perfect, i.e., if $P=P_{0}$. The inner loop is only active when there is a model uncertainty or other sources of uncertainties such as disturbances and sensor noises. Two assumptions are made in the problem formulation:

- The fault is non-repetitive.

- The disturbances are known or partially known

Thus $Q$ can be designed to robustify the feedback system. The proposed new controller design architecture has a clear separation between performance and robustness. The controller design methodology is as follows. A high performance robust system can be designed in two steps:

(a) Design $K_{0}=\tilde{V}^{-1} \tilde{U}$ to satisfy the system performance specifications with a nominal plant model $P_{0}$;

(b) Design $Q$ to satisfy the system robustness requirements. Note that the controller $Q$ will not affect the system nominal performance.

It should be emphasized that $K_{0}$ is not just any stabilizing controller as in most of controller parameterizations used in the literature, it is designed to satisfy certain performance specifications. For example, $K_{0}$ may be a simple $P I$ controller defined as $K_{0}=\frac{K_{p}(s+a)}{s}$, that satisfies our design specifications, in which case we can take $\tilde{U}=1$ and $\tilde{V}^{-1}=K_{0}=\frac{K_{p}(s+a)}{s}$

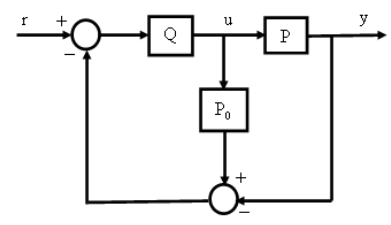

Fig. 3. Block diagram of Internal Model Control.

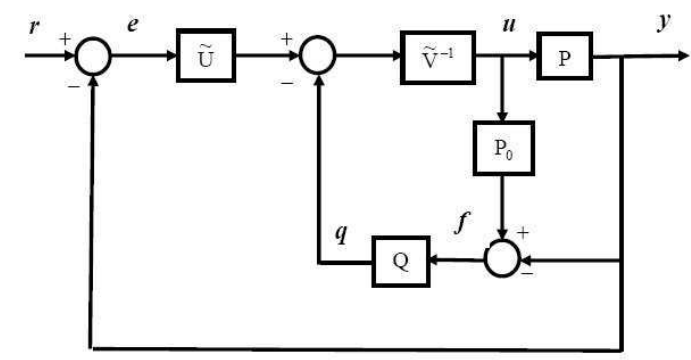

Fig. 4. FTC controller architecture.

The output error $f$ defined in Fig. 4 is the residual signal. In the fault diagnosis literature, $f$ is used to detect the possible faults in actuator and/or sensors. If $f=0$ we have not a fault but if $f \neq 0$ a robust controller is implemented using the standard feedback structure shown in Fig.1. The fault-tolerant controllers can be designed such that they provide adequate performance when there are no faults in the systems and as much tolerance as possible by any other fault-tolerant or robust controllers. Such controllers can be designed in two steps:

(a) Design $K_{0}=\tilde{V}^{-1} \tilde{U}$ to satisfy the performance for a nominal system;

(b) Design $Q$ to tolerate possible actuators and/or sensors failures (and model uncertainties).

The controller $Q$ can be synthesized using standard control strategies used in sensorized microgrippers, e.g., adaptive zero-phase error tracking controller [29], RST-based robust pole placement [30], robust feedforward-feedback control [31]. In our case we use the robust $H_{\infty}$ technique.

\section{B. Robustification}

We shall consider how to design the controller $Q$ for robustness. Consider the system described by the block 
diagram of the Fig.5.

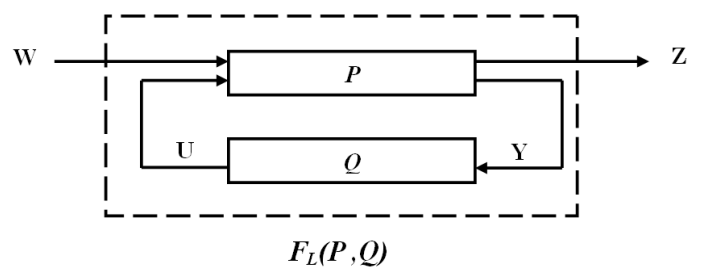

Fig. 5. $H_{\infty}$ robust control.

where $P$ is the generalized plant and $Q$ is the controller. Only finite-dimensional linear time invariant (LTI) systems and controllers will be considered in this paper. The generalized plant $P$ contains what is usually called the plant in a control problem plus all weighing functions. The signal $w$ contains all external inputs, including disturbances, sensor noise, and commands; the output $z$ is an error signal; $y$ is the measured variables; $u$ is the control input. The diagram is also referred to as a linear fractional transformation (LFT) on $Q$, and $P$ is called the coefficient matrix for the LFT. The resulting closed-loop transfer function from $w$ to $z$ is denoted by $T_{z w}$. The problem of $H_{\infty}$ standard is to synthesize a controller $Q$ which stabilizes the system $G$ and minimize the norm $H_{\infty}$ of $T_{z w}$ [32].

$$
P=\left[\begin{array}{ccc}
A & B_{1} & B_{2} \\
C_{1} & 0 & D_{12} \\
C_{2} & D_{21} & 0
\end{array}\right]
$$

The following assumptions are made:

1) $\left(A, B_{1}\right)$ is stabilizable and $\left(C_{1}, A\right)$ is detectable.

2) $\left(A, B_{2}\right)$ is stabilizable and $\left(C_{2}, A\right)$ is detectable.

3) $D_{12}^{\prime}\left[\begin{array}{ll}C_{1} & D_{12}\end{array}\right]=\left[\begin{array}{ll}0 & I\end{array}\right]$.

4) $\left[\begin{array}{ll}B_{1} & D_{21}\end{array}\right]^{T} D_{21}^{\prime}=\left[\begin{array}{ll}0 & I\end{array}\right]^{T}$.

The problem of $H_{\infty}$ standard is to synthesize a controller $Q$ which stabilizes the system $P$ and minimizes the $H_{\infty}$ norm of $\left\|T_{z w}\right\|_{\infty}$. Recall that the $H_{\infty}$ controller is :

$$
Q_{\infty}=\left[\begin{array}{cc}
\hat{A}_{\infty} \mid & -Z_{\infty} L_{\infty} \\
F_{\infty} & 0
\end{array}\right]
$$

$\hat{A}=A+\gamma^{-2} B_{1} B_{1}^{\prime} X_{\infty}+B_{2} F_{\infty} C_{2}$.

$F_{\infty}=-B_{2}^{\prime} X_{\infty}, L=-Y_{\infty} C_{2}, Z_{\infty}=\left(I-\gamma^{-2} Y_{\infty} X_{\infty}\right)^{-1}$. where $X_{\infty}=\operatorname{Ric}\left(H_{\infty}\right)$ and $Y_{\infty}=\operatorname{Ric}\left(J_{\infty}\right)$ the necessary and sufficient conditions for the existence of an admissible controller such that of $\left\|T_{z w}\right\|_{\infty}<\gamma$ are as follows:

1) $H_{\infty} \in \operatorname{dom}$ (Ric) and $X_{\infty}=\operatorname{Ric}\left(H_{\infty} \geq 0\right.$.

2) $J_{\infty} \in \operatorname{dom}($ Ric $)$ and $X_{\infty}=\operatorname{Ric}\left(H_{\infty} \geq 0\right.$.

3) $\rho\left(X_{\infty} Y_{\infty}\right)<\gamma^{2}$

The Hamiltonian matrices are defined as:

$$
\begin{gathered}
H_{\infty}=\left[\begin{array}{cc}
A & \gamma^{-2} B_{1} B_{1}^{T}-B_{2} B_{2}^{T} \\
-C_{1}^{T} C_{1} & -A^{T} \\
A^{T} & \gamma^{-2} C_{1} C_{1}^{T}-C_{2}^{T} C_{2} \\
-B_{1} B_{1}^{T} & -A
\end{array}\right] \\
J_{\infty}=
\end{gathered}
$$

\section{Robust $H_{\infty}$ Slave Controller}

The fault-tolerant control problem depends strongly on the type of faults that can appear in the system. In this paper, the faults are described as additive faults. In connection with FTC this might not be very useful. The reason is that the additive faults can be considered as external input signals to the system. External input signals will not cause any changes in the system dynamics. Specifically, they are not able to change the stability of the closed-loop system but the performance of the system will be affected. The controller $Q$ can be designed using the standard robust techniques as shown in Fig.6, where $\Delta$ include the additive fault.

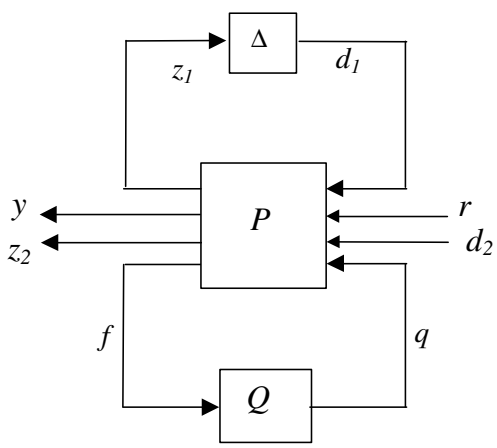

Fig. 6. The standard setup for design of $Q$ for systems with additive faults.

The $H_{\infty}$ design of $Q$ may be carried as per Fig. 6 thus:

$$
\begin{gathered}
z=\left[\begin{array}{c}
W_{1}(r-y) \\
W_{2} u
\end{array}\right], w=\left[\begin{array}{c}
r \\
d_{1} \\
d_{2}
\end{array}\right] \\
y=\left[\begin{array}{c}
r+W_{3} d_{1} \\
y+W_{4} d_{2}
\end{array}\right] \quad, \quad u=u_{s}
\end{gathered}
$$

To prove the passivity of overall wave-based bilateral micro-teleoperation system in Fig.1, we appropriately selected filters in order to passivate the communication channel with scaling factors (block 2). As it has been shown previously, the passivity of the slave part (block 3) connected to the $P I$ controller is ensured but it should be proved when considering the $H_{\infty}$ controller. Thus, the following condition $\left\|T_{z w}\right\|_{\infty}=1$ is added in order to ensure the passivity of the slave part.

\section{EXPERIMENTAl RESUlts AND Discussion}

To illustrate the behavior of the micro-teleoperation system and to confirm the findings of Section III, the FTC bilateral controller $K$ has been designed using the MATLAB function hinfsyn of the Robust Control Toolbox. Now, we will 
illustrate the validity of the proposed fault-tolerant control scaled telemanipulation system.

\section{A. Experimental Setup}

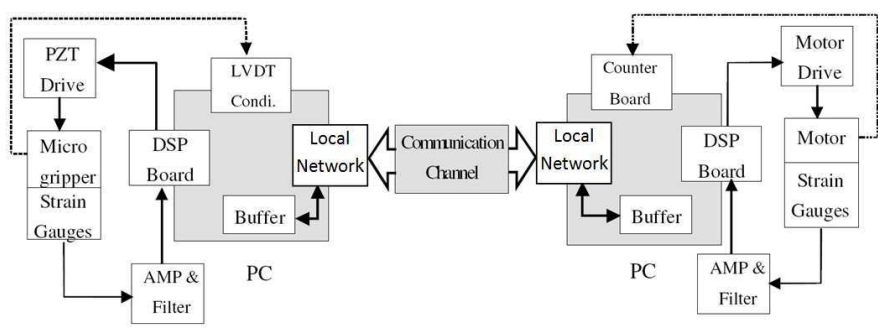

Fig. 7. Detailed description of the micro-teleoperation system.

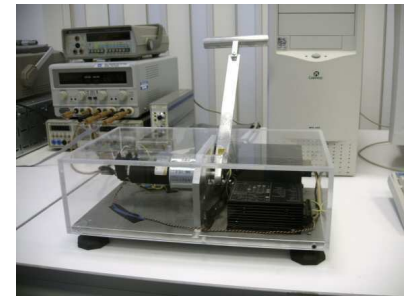

(a)

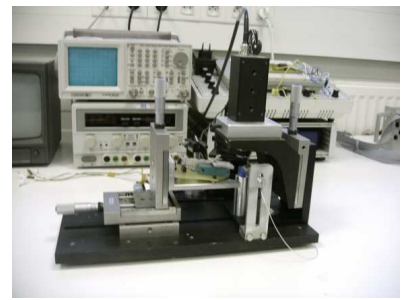

(b)
Fig. 8. ((b) The 1-dof haptic force feedback system (master) driven by a DC motor, and (c) the micromanipulation station under an optical microscope (slave) constituted by a 4-dof piezoelectric microgripper.

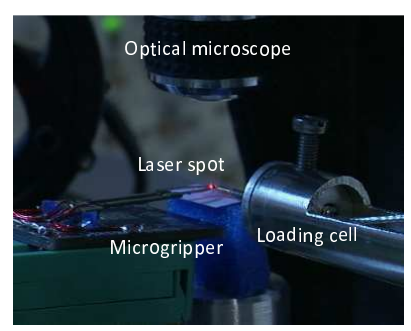

(a)

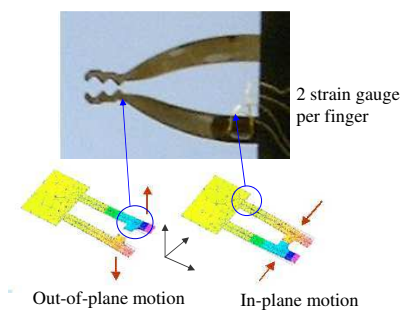

(b)
Fig. 9. Microprehensile Microrobot-On-Chip (MOC). (a) Structure of the two-fingered microprehensile MOC and (b) force sensorized end-effectors structure.

Fig. 7 and Fig. 8 show the developed experimental microteleoperation system. The system is made up of forcereflecting micromanipulator with a sensorized two-fingered microgripper operating under the field of view of an optical microscope. Figure 9(a) shows the micromanipulator with the four-degree-of-freedom (4-DOFs) microgripper used in the experiments. It is called MMOC (Microprehensile Microrobot-On-Chip) and has been developed at the Institut FEMTO-ST Besançon, France [33] operating under the field of view of an optical microscope. Thus the fingers are made from a monolithic micromachining approach since the microgripper, structure and actuators are built of the same piezoelectric zirconate titanate substrate. Each finger is constituted by a piezoelectric bimorph which is deflected in out-of-plane (z-axis) and/or in-plane (y-axis) directions in the bending mode. The proposed control algorithms are designed under the assumption that the environmental force in microenvironment is measurable but due to small size of the microgripper, it is difficult to incorporate strain gauge force sensors at the tip and scaling process amplifies greatly sensor noises to unacceptable level. The solution we selected consisted in using remote-located sensors, such as strain gauges, glued in the position of maximum strain of the gripper, as previously identified by FEM analysis (Fig.9(b)). A semiconductor strain gauge (type ESB-020-500 from Entran Devices) was glued to the flexure joint at both sides of the monolithic piezoelectric fingers. The circuit of strain gauges is arranged in the Wheatstone-bridge type with a special emphasis on compensation for thermal expansion and overlaying of normal strain. Sensor calibration for the bridge strain gauge is essential to determine the references of gripping force and tip displacement. In the first part, we measured the displacement of the force variation. Simultaneously, the signal of the strain gauge was measured to obtain the relationship between strain and displacement of the microgripper tip. The force sensor was calibrated by pushing the microgripper tip against the load cell (full scale: $100 \mathrm{mN}$, resolution: $0.05 \mathrm{mN}$ ). The slope of gripping force signal to strain gauge signal was approximately $78 \mu \mathrm{m} / \mathrm{V}$. The force sensibility is less than one milliNewton. Considering the statistics of calibration data, the maximum errors fitting line and data are $0.23 \mathrm{~V}$ in position sensor and $0.45 \mathrm{~V}$ in force sensor.The microgripper is currently attached to a $-x y z-$ micropositioning table which can be controlled via the local network by a force reflection interface at the operator side. The microgripper is controlled by a 1-dof master paddle with force feedback at the operator side (Fig.8a). The operator observes the microgripper position using a video device and the position is sensed by a high precision laser sensor with a resolution of $1 \mu \mathrm{m}$. The force sensitivity is less than one milliNewton. The paddle DC motor is controlled by the PWM amplifier, which operates in current control with the voltage given the D/A converter output of the I/O board. The force applied to the paddle lever, attached at the motor axis, is measured through the bending of the lever by a strain gauge bridge at the bottom of the lever with the strain bending amplified and converted by an A/D converter of the I/O board. The position of the lever is measured by an optical pulse incremental encoder on the motor axis. In order to experiment under time delays with various sizes, the communication unit that introduces bilateral time delays is simulated with a Matlab/Simulink block. The control loops and the model of the communication channel are composed of Matlab/Simulink block sets connected to DSP1103 boards. The operator can 


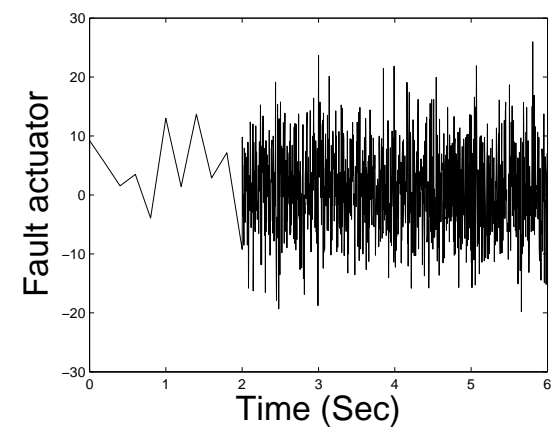

Fig. 10. Fault actuator.

choose on-line the scaling environment though a graphical user interface (GUI) and adjust by manual potentiometers the scaling parameters $\left(k_{p}, k_{f}\right)$ in real-time.

\section{B. Simulations and Experiments}

As mentioned before, the time-delay and motion and force scaling had been considered to violate the passivity condition. At first, we are going to show experimentally that the proposed scaling controller does not violate the total stability of the system for a human operator and a passive environment with unknown dynamics.

1) Simulation performances under microgripper faults: There is a number of reasons for using the FTC architecture. Using this architecture, the $Q$ parameter will be the FTC part of the controller. This means that the FTC part of the feedback controller is a modification of the existing slave controller. Thus, a slave controller change when a fault appears in the system is not a complete shift to another controller, but only a modification of the existing controller by adding a correction signal in the nominal controller, the $r$ signal in Fig.4.

For the $H_{\infty}$ slave controller design, the specifications are taken to ensure the position should track the master position delayed with the time $T$. The control $U$ should not exceed a pre-specified saturation limit and reject the fault.

The controller ensures the stability of the system with $\|f(G, Q)\|_{\infty}<\gamma$ for all $\|\Delta\|_{\infty}<1$. for $\gamma=1$, the passivity condition is guaranteed [35], so the weighting matrices are given by:

$$
\begin{aligned}
& W_{1}=0.019 \frac{s^{2}+10 s+10000}{s^{2}+10 s+0.1}, \quad W_{2}=0.5 \frac{s}{s+1000}, \quad W_{3}= \\
& 0.01 \frac{s}{0.001 s+1}, \quad W_{4}=0.001 .
\end{aligned}
$$

we synthesized the FTC architecture. The overall teleoperation system has been simulated with a sinusoidal reference and a fault actuator has been generated on the piezoelectric microgripper at $t=4 \mathrm{sec}$. In order to simulate external disturbances (degradation) due to electrical voltages variations, the fault is given as normally Gaussian distributed random signal with 10 mean value and a variance at 50 (see Fig.10). The results of the simulations are shown in Fig.12 and Fig.13.
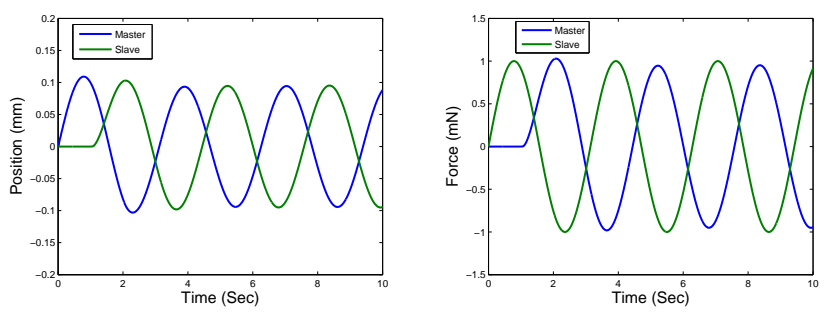

Fig. 11. Simulation results: sinusoidal position $\left(k_{p} x_{m}, x_{e}\right)$ and force $\left(f_{m}, k_{f} f_{e}\right)$ tracking for passive bilateral controller when $k_{p}=0.1$, $k_{f}=10$ and $T=1 \mathrm{Sec}$ without faults.
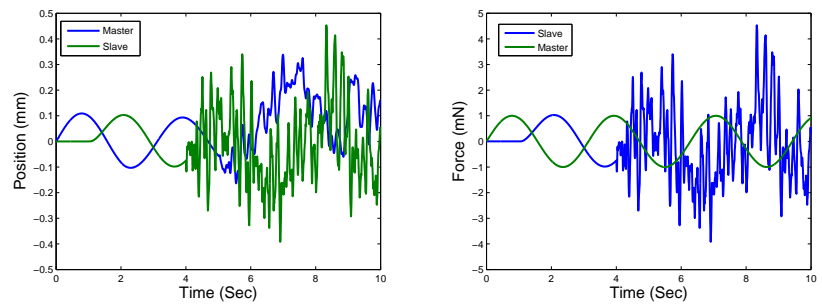

Fig. 12. Simulation results: sinusoidal position $\left(k_{p} x_{m}, x_{e}\right)$ and force $\left(f_{m}, k_{f} f_{e}\right)$ tracking for passive bilateral controller when $k_{p}=0.1$, $k_{f}=10$ and $T=1$ Sec with faults.
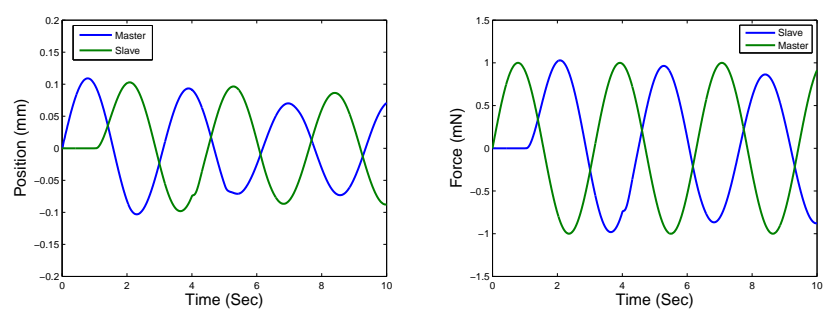

Fig. 13. Simulation results: sinusoidal position $\left(k_{p} x_{m}, x_{e}\right)$ and force $\left(f_{m}, k_{f} f_{e}\right)$ tracking with fault using FTC controller at $t=4 S e c$ when $k_{p}=0.1, k_{f}=10$ and $T=1 S e c$.
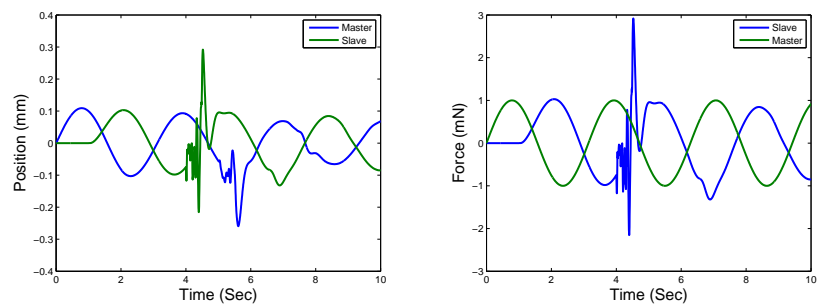

Fig. 14. Simulation results : sinusoidal position $\left(k_{p} x_{m}, x_{e}\right)$ and force $\left(f_{m}, k_{f} f_{e}\right)$ tracking with fault using FTC controller at $t=4.5 \mathrm{Sec}$ when $k_{p}=0.1, k_{f}=10$ and $T=1 \mathrm{Sec}$. 


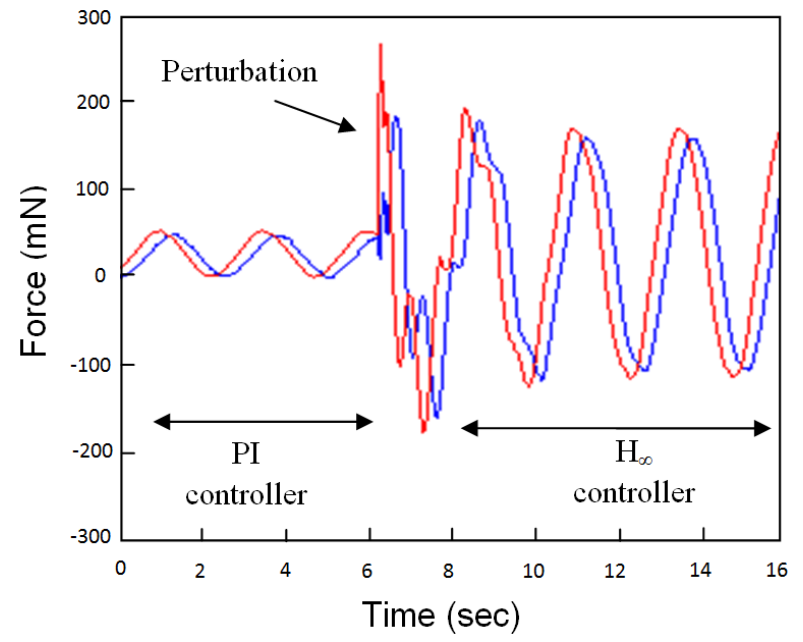

Fig. 15. Transient response of the microgripper using FTC feedback when subjected to a burst disturbance when $k_{p}=0.1, k_{f}=10$ and $T=1 \mathrm{Sec}$.
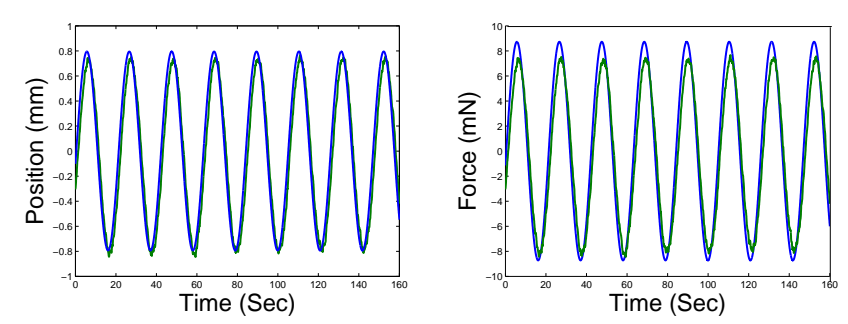

Fig. 16. Experimental results: sinusoidal position $\left(k_{p} x_{m}, x_{e}\right)$ and force $\left(f_{m}, k_{f} f_{e}\right)$ tracking for passive bilateral controller when $k_{p}=0.1, k_{f}=$ 10 and $T=1 \mathrm{Sec}$ without fault.

Simulation results of the micro-teleoperation system with faulty actuator is shown in Fig.12. It can be seen directly from this figure a bad performance with lot of noise, so the controller $Q$ needs to be included. As calculated above, the robust controller $Q$ can be applied for the faulty closed loop system. In this example, the $Q$ controller is implemented with a switching system. Based on the design of $Q$, the simulation results of the micro-teleoperation system are shown in Fig.13 and Fig.14. In Fig.13, the piezoelectric microgripper actuators is subjected to a voltage leakage and in Fig.14, a sudden mechanical fault occurs at the microgripper's fingers. The standard robust controller is independent of the nominal controller $P I$. In the worst case (i.e., when the uncertainties are in the worst case), our controller implementation will be equivalent to the existing robust control design. Of course, if there is no uncertainty, our controller will perform as well as a nominal controller does.

2) Experimental validation of FTC controller: Experimental results have been carried out. In a given environment of micromanipulation, the operator can choose online the couple of scaling parameters $\left(k_{p}, k_{f}\right)$ by adjusting their values in
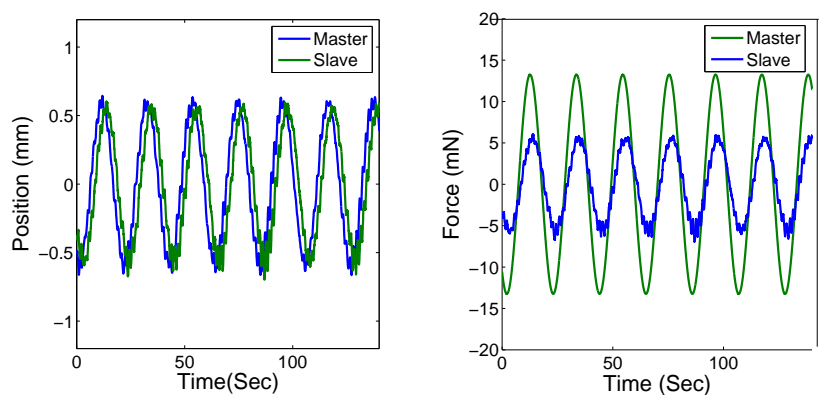

Fig. 17. Experimental results: sinusoidal position $\left(k_{p} x_{m}, x_{e}\right)$ and force $\left(f_{m}, k_{f} f_{e}\right)$ tracking for $H_{\infty}$ bilateral controller when $k_{p}=0.1, k_{f}=10$ and $T=1 \mathrm{Sec}$ with fault.

the range of lower and upper bounds. Assuming an exact and linear time-invariant model of the environment at the microscopic scale is generally a tenuous presumption due to nonlinear effects. Since the detected force is of very small magnitude, major position and force amplification should be made following the nature of the objects (fragile, soft, rigid) and the nature of the nonlinear interaction (structurallydominated or surface-dominated interaction). In this study, the microenvironment is typically modeled mainly as a spring system (neglecting the small damper and inertial object) $: Z_{e} \in\left(\frac{k_{e}}{s}+b_{e}\right): b_{e}=0.05 ; 500 \leq k_{e} \leq 1500$. It corresponds to typical teleoperated "pick-and-place" operations in serial microassembly tasks of complex 3-D microelectromechanical systems (MEMS) [34]. It necessitates the detection and control of interaction forces in a wide dynamic range from few microNewtons to hundreds of milliNewtons.

The master and slave position and force tracking profiles are illustrated in Fig.16 when considering a soft contact with corresponding scaling factors $k_{p}=N=0.1$ and $k_{f}=1 / N=10$ and a constant time-delay $T=1 \mathrm{sec}$ (passivity condition). As stated previously, the microgripper is required to work in uncertain environments unlike most MEMS micromanipulation tasks where significant changes occur according to task. Many unpredictable factors, such as vibration disturbances, adhesive microforces, calibration errors, image processing errors lead to microgripping failure. To simplify the study, we suppose that the parameters of environment dynamics are known to the extent that they lie in some intervals, that is, uncertain parameters vary over ranges about nominal values. In these cases, both $P I$ and $H_{\infty}$ optimization controllers fails in ensuring stable performances.

As illustration of the high-performances and robustness characteristics of the FTC controller, the force transient state has been experimentally studied in two specific cases: (i) a burst disturbance (see Fig.15.a) and (ii) a smooth step damping (see Fig.15.b) produced mechanically on the microgripper's fingers. Both cases show good performances in terms of robustness against external perturbations, stable 
characteristics with fast convergence time and good force transparency performances. In a permanent regime, Fig.16 and Fig.17 demonstrate the long term performances when normal operating conditions are applied (without fault) leading to passivity conditions involving the $P I$ slave controller and when faulty conditions are detected. These results demonstrate that the wave variables-based passivity approach ensures good transparency performances ( $P I$ controller) and important robustness $\left(H_{\infty}\right.$ controller) in the presence of various faults. A good agreement between simulation and experimental results is found. In fact, our framework provides a great flexibility in controller design, for example, one could still use all the robust and $H_{\infty}$ design techniques here. All one has to do is to start with a good performance controller and then every thing can proceed as in the standard robust control design procedure to find the robust controller $Q$. The only difference is that we are not interested in plugging $Q$ into the controller parameterization to find the total controller rather we will implement the performance controller and the robust controller $Q$ separately.

\section{CONCLUSION}

In this paper, we have proposed the design of a bilateral controller for micro teleoperation system and an architecture for fault tolerant control has been used for a 4-dof piezoelectric microgripper. The bilateral controller ensures the passivity of the system and take into account the communication delays, the variation of the micro-environment as well as the scaling factors. For the slave part, applying the Youla parameterization structure an additional controller has been introduced as the main tool to achieve fault tolerance. A feature of the FTC structure is that it automatically includes a diagnostic signal. The presented simulation and experimental results show that the FTC controller provide adequate performance when there are no faults in the system and a high degree of tolerance by using $H_{\infty}$ robust controller.

\section{REFERENCES}

[1] Anderson, R. J. and Spong, M.W., "Bilateral Control of Teleoperators with Time Delay." IEEE Transactions on Automatic Control, Vol. 34, No. 5, pp. 494-501, 1989.

[2] Niemeyer, G. and Slotine, J. E., "Stable Adaptive Teleoperation." IEEE Journal of Oceanic Engineering, Vol. 16, No. 1, pp. 152-162, 1992.

[3] Park K ,Chung W.K and Youn Y., "Obtaining passivity of microteleoperation handling a small inertia object" Proc. Of IEEE Int. Conf. on Robotics and Automation, pp.3266-3271, 2002.

[4] Hashitrudi-Zaad K., Salcudean S.E., "On the Use of Local Force Feedback for Transparent Teleoperation", IEEE Int. Conf. on Robotics and Automation, pp.1863-1869, 1999.

[5] A. Bolopion, H. Xie, D. Sinan Haliyo, S. Régnier, "Haptic teleoperation for 3D microassembly of spherical objects", IEEE/ASME Transactions on Mechatronics, 2010, in press, DOI:10.1109/TMECH.2010.2090892.

[6] C.D. Onal and M. Sitti, "A scaled bilateral control system for experimental one-dimensional teleoperated nanomanipulation", International Journal of Robotics Research, Vol.28, No.4, pp.484-497, 2009.
[7] M. Boukhnifer, A. Ferreira, "Passive bilateral control of teleoperators under time-delay and scaling factors", 44th IEEE Conference on Decision and Control, and the European Control Conference, Seville, Spain, Dec. 12-15, pp.6972-6977, 2005.

[8] M. Sitti, H. Hashimoto, "Teleoperated touch feedback of surfaces at the nanoscale: modeling and experiments", IEEE/ASME Transactions on Mechatronics, Vol.8, No.2, pp.287-298, June 2003.

[9] M. Savia, H. Koivo, "Contact micromanipulation - survey of strategies", IEEE/ASME Transactions on Mechatronics, Vol.14, No.4, pp.504-514, June 2009.

[10] M. Boukhnifer, A. Ferreira, "Wave-based passive control for transparent micro-teleoperation system", Robotics and Autonomous Systems, Vol.54, pp.601-615, 2006.

[11] Zhou, K. and Ren, Z., "A new controller architecture for high performance robust, and fault-tolerant control", IEEE Transactions on Automatic Control, Vol. 46(10), 2001, pp. 16131618.

[12] Jiang J., Zhang Y., "Accepting performance degradation in faulttolerant control system design", IEEE Transactions on Control, Systems Technology, Vol.14, N.2, March 2006, pp.284-292.

[13] Karpenko M., Sepehri N., "Robust position control of an electrohydraulic actuator with a faulty actuator piston seal", ASME Journal of Dynamic Systems, Measurement and Control, Vol. 125, Sept.2003, pp.413-423.

[14] Lu H-C, Lin W-C.,"Robust controller with disturbance rejection for hydraulic servo systems", IEEE Transactions on Industrial Electronics, Vol.40, N.1, Feb.1993, pp.157-161.

[15] Edwards C., Tan C-P,'Sensor fault tolerant control using sliding mode observers", IFAC Control Enginnering Practice, Vol.14, 2006, pp.897908.

[16] M. Mohammadzaheri, L. Chen, "A critical review of the most popular types of neuro control", Asian Journal of Control, Volume 14, Issue 1, pages 1-11, January 2012

[17] Yu, W. W., G. R. Chen, and J. D. Cao, "Adaptive synchronization of uncertain coupled stochastic complex networks," Asian Journal of Control, Vol. 13, No. 3, pp. 418-429, 2011.

[18] C. Spelta, S.M. Savaresi, F.Codec, M. Montiglio, M. Ieluzzi, "SmartBogie: Semi-Active Lateral Control of Railway Vehicles, Asian Journal of Control, 25 January 2012.

[19] X-H. Chang, G-H. Yang, "Fuzzy robust constrained model predictive control for nonlinear systems", Asian Journal of Control, Volume 13, Issue 6, pages 947-955, November 2011

[20] Stoustrup, J. and Niemann, H.,'Fault tolerant feedback control using the Youla parameterization", Proceedings of the $6^{\text {th }}$ European Control Conference, Porto, Portugal, Sept. 2001, pp.19701974.

[21] C.A. Desoer, M. Vidyasagar, 'Feedback Systems: Input-Output Properties", Academic, New York, 1975.

[22] R.J. Anderson, W.J. Spong, "Asymptotic stability for force reflecting teleoperators with time delay", International Journal of Robotic Research, 11 (2) (1992) 135149.

[23] Y. Yokohoji, T. Yoshikawa, "Bilateral control of master-slave manipulators for ideal kinesthetic coupling-formulation and experiment", IEEE Transactions on Robotics and Automation, 10 (1994) 605620.

[24] G. Niemeyer, J.J. Slotine, "Using wave variables for system analysis and robot control", IEEE International Conference on Robotics and Automation, Albuquerque, New Mexico, 1997, pp. 16191625.

[25] Niemeyer, G., "Using Wave Variables in Time Delayed Force Reflecting Teleoperation", Thesis PhD, Department of Aeronautics and Astronautics, Massachusetts Institute of Technology, 1996.

[26] Murakami E.A., Yamada T., Kondo T., Ito K., "Performance Evaluation of Bilateral Micro-Teleoperation Systems based on Man-Machine Dynamic Characteristics", Int. Conf. on SICE, Aug. 5-7, Osaka, Japan, pp.2941-2946, 2002.

[27] M. Boukhnifer, A. Ferreira, " $H_{\infty}$ Loop Shaping Bilateral Controller for a Two-Fingered Tele-Micromanipulation System", IEEE Transactions on Control Systems Technology, Vol.15, No.5, pp. 891-905, 2007.

[28] M. Morari and E. Zafiriou, "Robust Process Control". Upper Saddle River, NJ: Prentice-Hall, 1989.

[29] J. Park, S. Kim, D-H. Kim, B. Kim, S.J. Kwon, J-O. Park, KI Lee, "Identification and control of a sensorized microgripper for 
micromanipulation", IEEE/ASME Transactions on Mechatronics, VOL. 10(5), 2005, pp.601-606.

[30] Grossard, M. Boukallel, M. Chaillet, N. Rotinat-Libersa, C., "Modeling and Robust Control Strategy for a Control-Optimized Piezoelectric Microgripper ", IEEE/ASME Transactions on Mechatronics, 2010, in press, 10.1109/TMECH.2010.2050146.

[31] Rakotondrabe M., Rabenorosoa K., Agnus J. and Chaillet N., "Robust feedforward-feedback control of a nonlinear and oscillating 2dof piezocantilever", IEEE Transactions on Automation Science and Engineering, 2010, in press, DOI.10.1109/TASE.2010.2099218.

[32] J. Doyle, K. Glover, P. Khargonekar, and B. Francis, "State-space solutions to standard $\mathrm{H}_{2}$ and $\mathrm{H}_{\infty}$ control problems", IEEE Transactions on Automatic Control, AC-34, no. 8, pp. 831-847, Aug. 1989.

[33] A. Ferreira, J. Agnus, N. Chaillet, J.M. Breguet, "A Smart Microrobot On Chip: Design, Identification and Control", IEEE/ASME Transactions on Mechatronics, Vol.9, No.3, September 2004, pp.508-519.

[34] N. Ando, P. Korondi, H. Hashimoto, "Networked Telemicromanipulation Systems "Haptic Loupe", IEEE Transactions on Industrial Electronics, vol 51, No 6, Dec 2004, pp.1259-1271.

[35] Petros A. Ioannou and Jing sun, "Robust Adaptive Control", Prentice Hall,1996. 